Conclusion Patients with PID had a high rate of PICU admission. Multi-organ failure was associated with mortality. Despite adequte support mortality rates were around $50 \%$.

\section{PULMONARY HAEMORRHAGE IN VERY LOW BIRTH WEIGHT INFANTS: 10 YEARS EXPERIENCE IN TERTIARY NICU}

doi:10.1136/archdischild-2012-302724.0816

${ }^{1} \mathrm{~A}$ Nosherwan, ${ }^{1} \mathrm{~K}$ Bermijo, 'D O'Leary, 1,2,3E Molloy. 'Neonatology, National Maternity Hospital; ${ }^{2}$ Paediatrics, University College Dublin; ${ }^{3}$ Paediatrics, Royal College of Surgeons in Ireland, Dublin, Ireland

Aims To study the clinical course, associated neonatal morbidity and mortality following pulmonary haemorrhage $(\mathrm{PH})$ in VLBW infants.

Methods Infants $<1500$ gms that developed pulmonary haemorrhage were identified from the hospital database. Outcome was measured as death or survival following acute $\mathrm{PH}$.

Results Total of 1206 VLBW infants were admitted in our NICU between 2002 to 2011. Incidence of $\mathrm{PH}$ was 3\%(n=40) in this group. Mean gestational age (SD) was $26 \pm 1.8$ weeks with mean Birth weight of $0.8 \pm 0.2 \mathrm{~kg}$. Cause of preterm delivery was fetal distress $n=7$, maternal PET $n=8$, maternal sepsis $n=10$ and preterm labour $n=10$. Eleven of those mothers received antenatal steroids. Antenatal steroids were associated with improved survival $(p<0.001)$. Eight babies had evidence of intrauterine growth retardation and six babies were septic at birth. Five patients required cardiopulmonary resuscitation at birth. The mean age at $\mathrm{PH}$ was $47.7 \pm 28$ hours. Sixteen patients received prophylactic surfactant. Two patients had mild, nine had moderate and twenty-nine had severe $\mathrm{PH}$. Ten cases were treated with surfactant with acute PH. Half of the infants had a PDA. Eighteen patients were coagulopathic at the time of $\mathrm{PH}$. Twenty-one babies died (53\%) and none had received antenatal steroids. In the surviving infants, ten developed CLD and six had Mental Developmental Index $<70$.

Conclusion Our institutional incidence of pulmonary haemorrhage and outcome is similar to other reports in the literature. Comprehensive evidence base management strategies are required to standardise care of the VLBW infants who develop $\mathrm{PH}$.

\section{EARLY NEONATAL OUTCOME IN NEWBORNS EXPOSED TO CHEMOTHERAPY DURING FETAL PERIOD}

doi:10.1136/archdischild-2012-302724.0817

'M Fumagalli, 'L Bassi, ${ }^{2} \mathrm{G}$ Scarfone, ${ }^{2} \mathrm{~F}$ Peccatori, 'S Pisoni, 'S Passera, 'M Groppo, 'A De Carli, 'I Sirgiovanni, 'F Dessimone, 'F Mosca. 'NICU, Fondazione IRCCS Ca' Granda, Ospedale Maggiore Policlinico University of Milan; ${ }^{2}$ Second Obstetric and Gynecologic Clinic, University of Milan and Fondazione IRCCS Ca' Granda Ospedale Maggiore Policlinico, Milan, Italy
Introduction Cancer treatment in pregnant women is still a matter of debate as life-saving therapies for the mother and adverse effects for the fetus. Recent data support that chemotherapy from 14 wks GA onwards as safe in terms of early neonatal and long-term outcomes in children, while impaired neurodevelopmental outcome appear to be related to prematurity.

Aim To describe the early neonatal outcome in newborns exposed to chemotherapy during pregnancy.

Methods Clinical data of inborn babies, born between 2005 and 2011, from mother treated with chemotherapy, were retrospectively collected from clinical notes.

Results In our population of neonates exposed to chemotherapy during pregnancy 18\% (4/22) were born between 25 and 33 weeks of GA and 73\% (16/22) between 34 and 36 wks, 9\% at term, $(2 / 22)$. The $70 \%$ was admitted to post-natal ward and the $95 \%$ was AGA, while only $5 \%$ SGA. Neonatal complications observed were: respiratory distress syndrome 32\% (7/22; 3/7 CPAP, 3/7 meccanical ventilation), hypoglycemia $23 \%(5 / 22)$ and jaundice $23 \%(5 / 22)$. Two very preterm babies $(<27 \mathrm{wks})$ developed intraventricular haemorrhage. Not cardiac complications and not significant hematological abnormalities (neutropenia, severe acute anemia and low platelet count) were observed in the neonatal period.

Conclusions According to our data chemotherapy during pregnancy doesn't seem to influence early neonatal outcome. Otherwise more studies are needed to confirm the safety of cancer treatment during pregnancy in terms of long-term cognitive and neurobehavioural outcomes in order to define treatment strategies aimed to reduce iatrogenic preterm birth.

\section{LENGTH OF HOSPITAL STAY (LOS) OF NEONATES UNDERGOING SURGERY AT A TERTIARY NEONATAL UNIT}

doi:10.1136/archdischild-2012-302724.0818

${ }^{1}$ SK Shetty, 'N Kennea, ${ }^{2}$ S Giuliani, 'P Desai, '1 Richards. 'Neonatal Unit; ${ }^{2}$ Paediatric Surgery Unit, St George's Hospital NHS Trust, London, UK

Background and Aims Detailed length of stay (LOS) data for infants following surgical procedures are important for prediction of neonatal resources and helpful when counselling parents following a new diagnosis. There are few recent data available on LOS for different surgical conditions in the literature, and none describing a range of procedures from one centre.

Objective To describe LOS for common surgical procedures in a single centre tertiary referral neonatal unit.

Methods Data were collected for a 5-year period. If babies were transferred to another unit following surgery, these units were contacted to determine total LOS. We included babies that had surgery during their first admission to our unit and who survived to discharge.

Results 